\title{
INFINITELY MANY SIGN-CHANGING SOLUTIONS FOR QUASILINEAR SCHRÖDINGER EQUATIONS IN $\mathbb{R}^{N *}$
}

\author{
YINBIN DENG ${ }^{\dagger}$, SHUANGJIE PENG ${ }^{\ddagger}$, AND JIXIU WANG ${ }^{\S}$
}

\begin{abstract}
This paper is concerned with constructing radial solutions with arbitrarily many sign changes for quasilinear Schrödinger equations in $\mathbb{R}^{N}$ which have appeared as several models in mathematical physics. For any given integer $k \geq 0$, by using a minimization argument, we obtain a sign-changing minimizer with $k$ nodes of a minimization problem with double constraints, and by applying an energy comparison method we prove that the minimizer is indeed a solution of the quasilinear Schrödinger equation.
\end{abstract}

Key words. Minimization problem, quasilinear Schrödinger equations, radial solutions, signchanging solutions.

AMS subject classifications. 35J10, 35P30, 35Q55, 58E40, 58J32.

\section{Introduction}

This paper has been motivated by the quasilinear Schrödinger equations

$$
i \partial_{t} z=-\Delta z+W(x) z-f\left(|z|^{2}\right) z-\kappa \Delta h\left(|z|^{2}\right) h^{\prime}\left(|z|^{2}\right) z,
$$

where $z: \mathbb{R} \times \mathbb{R}^{N} \rightarrow \mathbb{C}, W: \mathbb{R}^{N} \rightarrow \mathbb{R}$ is a given potential, $\kappa$ is a real constant, and $f, h$ are real functions of essentially pure power forms.

The semilinear case corresponding to $\kappa=0$ has been studied extensively in recent years; for example, see Berestycki and Lions [7], Floer and Weinstein [17], Rabinowitz [34], and Strauss [36]. Quasilinear equations of form (1.1) appear more naturally in mathematical physics and have been derived as models of several physical phenomena corresponding to various types of $h$. For instance, the case $h(s)=s$ models the time evolution of the condensate wave function in super-fluid film ([21, 22]). This equation has been called the superfluid film equation in fluid mechanics by Kurihara [21]. In the case $h(s)=(1+s)^{1 / 2}$, problem (1.1) models the self-channeling of a high-power ultra short laser in matter, the propagation of a high-irradiance laser in a plasma creates an optical index depending nonlinearly on the light intensity and this leads to an interesting new nonlinear wave equation (see $[8,12,16,35])$. Problem (1.1) also appears in plasma physics and fluid mechanics [4, 20, 33], in mechanics [19], in the theory of Heisenberg ferromagnets and magnons [4] and in condensed matter theory [28]. For more physical motivations and more references dealing with applications, we can refer Brüll and Lange [10], Lange et al. [23], Poppenberg et al. [32], and references therein.

In the mathematical literature, very few results are known on equations of the form (1.1). In the case $h(s)=(1+s)^{1 / 2}$ the local well posedness is proved in [16] for the space dimension $N=1,2,3$ where smallness assumptions on the initial value are needed if $N=2,3$. The case $h(s)=s, N=1$ is investigated in [23], and the case of

${ }^{*}$ Received: September 21, 2010; accepted (in revised version): January 10, 2011. Communicated by Jack Xin.

${ }^{\dagger}$ School of Mathematics and Statistics, Huazhong Normal University, Wuhan, 430079, P.R. China (ybdeng@mail.ccnu.edu.cn).

${ }^{\ddagger}$ School of Mathematics and Statistics, Huazhong Normal University, Wuhan, 430079, P.R. China (sjpeng@mail.ccnu.edu.cn).

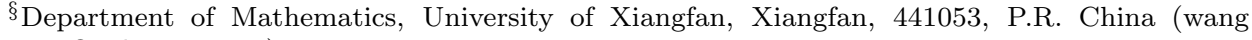
jixiu127@yahoo.com.cn). 
general $h(s)$ is considered in [30] for $N=1$ and in [31] for arbitrary space dimension $N \geq 1$.

Here we focus on the case $h(s)=s, \kappa=1 / 2$. It was shown that a system describing the self-trapped electron on a lattice can be reduced in the continuum limit to this case, and numerical results on this equation have been given in [9]. Our special interest is the existence of standing waves, that is, solutions of type $z(t, x)=\exp (-i E t) u(x)$, where $E \in \mathbb{R}$ and $u$ is a real function. It is well known that $z(t, x)$ satisfies (1.1) if and only if the function $u(x)$ solves the following equation

$$
\left\{\begin{array}{l}
-\Delta u+V(x) u-\frac{1}{2} \Delta\left(u^{2}\right) u=f\left(u^{2}\right) u, \quad x \in \mathbb{R}^{N}, \\
u \rightarrow 0, \quad \text { as }|x| \rightarrow \infty,
\end{array}\right.
$$

where $V(x)=W(x)-E$ is a new potential.

The existence of a positive ground state solution of problem (1.2) has been proved in Poppenberg et al. [32] and Liu and Wang [24] by using a constrained minimization argument, which gives a solution with an unknown Lagrange multiplier $\lambda$ in front of the nonlinear term. In Liu et al. [25], by a change of variables the quasilinear problem was transformed to a semilinear one and an Orlitz space framework was used as the working space, and the existence of a positive solution of problem (1.2) for any prescribed $\lambda>0$ was proved by using the Mountain-Pass theorem (e.g., Ambrosetti and Rabinowitz [1]). The same method of changing variables was also used recently to obtain positive solutions of problem (1.2) in [13] for the case of subcritical growth and in [5] for the case of critical growth. Along this line, one could also look for signchanging solutions (as, for example, in [26]) by utilizing the Nehari method; Liu et al. treated more general quasilinear problems and obtained positive and sign-changing solutions. The main mathematical difficulties with problem (1.2) are caused by the nonlinearity involving second order space derivatives. In the variational formulation, these difficulties concern the nonlinear functional $\Psi(u)=\int_{\mathbb{R}^{N}} u^{2}|\nabla u|^{2}$ which is homogeneous of order 4 and non-convex. A further problem is caused by the usual lack of compactness since these problems are dealt with in the whole $\mathbb{R}^{N}$.

We remark that the solutions given in the above papers were obtained mainly by using a constrained minimization method or the Mountain-Pass theorem and hence are ground state solutions. Generally, these types of solutions are orbitally stable, since they have the least energy (i.e. the mountain-pass level $c$ ) among all solutions. However, concerning sign-changing solutions, we found no results except in [26] where the solutions change sign exactly one time. Physically speaking, a sign-changing solution $u$ of (1.2) corresponds an excited standing wave $z(t, x)=\exp (-i E t) u(x)$ of (1.1) (see [15]), which generally has less orbital stability since a sign-changing solution has at least double the least energy $c$. In this paper, we construct radial solutions of (1.2) with arbitrarily many sign changes. When $\kappa=0$ in (1.1), the existence of radial sign-changing solutions has been explored thoroughly; we refer the readers to $[2,11,14,37]$ and the references therein. In dimensions $N=4$ and $N \geq 6$, Bartsch and Willem [3] were able to construct sequences of nonradial sign-changing solutions of (1.1). Concerning nonradial positive solutions with higher energy of (1.2), we should point out that a recent paper [27] gives a very interesting result: on the annulus $\left\{x \in \mathbb{R}^{N}: a \leq|x| \leq a+1\right\}$, (1.2) has a sequence of nonradial positive solutions with higher energy for large $a$.

We will construct infinitely many solutions to problem (1.2) by the Nehari method. To emphasize our main idea, we only concentrate on the case where $f(s)$ is purely in 
power form; that is, we consider

$$
\left\{\begin{array}{l}
-\Delta u+V(x) u-\frac{1}{2} \Delta\left(|u|^{2}\right) u=\lambda|u|^{p-2} u, \quad x \in \mathbb{R}^{N}, \\
u \rightarrow 0, \quad \text { as }|x| \rightarrow \infty,
\end{array}\right.
$$

where $\lambda>0, N \geq 2,4<p<22^{*}, 2^{*}$ is the Sobolev critical exponent, that is, $2^{*}=$ $2 N /(N-2)$ if $N \geq 3$ and $2^{*}=+\infty$ if $N=2$.

We assume $V(x)$ is a radially symmetric function and satisfies

$(\mathrm{V}): V \in C\left(\mathbb{R}^{N}, \mathbb{R}\right), 0<V_{0}:=\inf _{\mathbb{R}^{N}} V(x)$.

Our main result for (1.3) is:

TheOREm 1.1. Suppose that $V(x)$ satisfies $(V), 4<p<22^{*}, \lambda>0$. Then for any $k \in\{0,1,2, \cdots\}$ there exists a pair of radial solutions $u_{k}^{ \pm}$of (1.3) with the following properties:

(i) $u_{k}^{-}(0)<0<u_{k}^{+}(0)$;

(ii) $u_{k}^{ \pm}$possess exactly $k$ nodes $r_{i}$ with $0<r_{1}<r_{2}<\cdots<r_{k}<+\infty$, and $\left.u_{k}^{ \pm}(x)\right|_{|x|=r_{i}}=0, i=1,2, \cdots, k$.

On the main result, we give some remarks.

$u_{k}^{ \pm}$have at least the energy $(k+1) c$ and hence belong to higher energy solutions if $k \geq 1$, where $c$ is the least energy corresponding to (1.3). Since $|u|^{p-2} u$ is odd in $u$, we see that $-u_{k}^{+}$and $-u_{k}^{-}$are also sign-changing solutions to (1.3). However, we can not claim that $u_{k}^{+}=-u_{k}^{-}$since the nodes $r_{1}, \cdots, r_{k}$ might not be unique. It is a very interesting problem to study the uniqueness of $k$-node solutions (up to a sign) of (1.3) for given $k \in \mathbb{N}$ even $\kappa=0$.

In Theorem 1.1, we mainly deal with the nonlinear term $|u|^{p-2} u$. But, the oddness assumption on nonlinear term is actually unnecessary. Our main result holds true for general nonlinearity $f(|x|, u)$ with properties similar to those in [2] or [11]. Indeed, in this case we only need to extend $f(|x|, u)$ as follows:

$$
f^{+}(|x|, u):=\left\{\begin{array}{l}
f(|x|, u), \text { if } u \geq 0, \\
-f(|x|,-u), \text { if } u<0,
\end{array}\right.
$$

or

$$
f^{-}(|x|, u):=\left\{\begin{array}{l}
-f(|x|,-u), \text { if } u \geq 0, \\
f(|x|, u), \text { if } u<0,
\end{array}\right.
$$

and define $J^{ \pm}(u), c_{k}^{ \pm}=\inf _{u \in M_{k^{ \pm}}^{ \pm}} J^{ \pm}(u)$ in the same way as those in [11]. By a similar argument, we can prove that $c_{k}^{ \pm}$can be attained by $u_{k}^{ \pm}$, which must be the $k$-node solutions for the corresponding problem.

Particularly, we can prove the following Corollary.

Corollary 1.1. Suppose that $V(x)$ satisfies $(V), 4<p, q<22^{*}, \lambda, \mu>0$. Then for every integer $k$ there exists a pair $u_{k}^{ \pm}$of radial solutions of

$$
\left\{\begin{array}{l}
-\Delta u+V(x) u-\frac{1}{2} \Delta\left(|u|^{2}\right) u=\lambda u_{+}{ }^{p-1}-\mu u_{-}^{q-1}, \quad x \in \mathbb{R}^{N}, \\
u \rightarrow 0, \quad \text { as }|x| \rightarrow \infty,
\end{array}\right.
$$

with $u_{k}^{-}(0)<0<u_{k}^{+}(0)$, having exactly $k$ nodes. 
Since $22^{*}=4 N /(N-2)$ behaves like a critical exponent for equation, by using a Pohozaev type variational identity, Liu et al. [26] proved that (1.3) has no positive solutions in $H^{1}\left(\mathbb{R}^{N}\right)$ with $\int_{\mathbb{R}^{N}} u^{2}|\nabla u|^{2}<\infty$ if $p \geq 22^{*}$. A natural problem is whether or not (1.3) has sign-changing solutions (including radial solutions) if $p \geq 22^{*}$. We will give some results on the problem in a future work.

In $[5,13,24,26,32]$, to obtain ground state solutions of $(1.3), V(x)$ should satisfy

$$
0<V_{0}=\inf _{\mathbb{R}^{N}} V(x) \leq V_{\infty}=\lim _{|x| \rightarrow \infty} V(x) .
$$

Hence, another interesting problem is whether or not ground state solutions or higher energy solutions exist if (1.4) is not satisfied?

We will prove Theorem 1.1 by looking for a minimizer for a constrained minimization problem in a special space in which each function changes sign $k(k \in\{0,1,2, \cdots\})$ times, and then verify that the minimizer is smooth and indeed a solution to (1.3) by analyzing the least energy related to the minimizer. Unlike those done in the above mentioned papers, we will work directly with the functional $I$ corresponding to (1.3) in spite of its lack of smoothness. We mention here that the main method to prove our theorem was introduced by Bartsch and Willem in [2] and Cao and Zhu in [11] independently, but, as we can see later, the appearance of the quasilinear operator $\Delta\left(\cdot{ }^{2}\right)$. may cause more difficulties.

The paper is organized as follows: Section 2 is devoted to preliminaries and some useful lemmas. Theorem 1.1 will be proved in Section 3 .

\section{Some preliminary lemmas}

In this section, we give some definitions and lemmas. The proof of some lemmas mentioned here can be found in the corresponding references. Here we use $|u|_{q}$ to denote the $L^{q}\left(\mathbb{R}^{N}\right)$ norm. In the following, we set

$$
H_{r}^{1}\left(\mathbb{R}^{N}\right)=\left\{u \in H^{1}\left(\mathbb{R}^{N}\right): u(x)=u(|x|)\right\},
$$

and

$$
X=\left\{\left.u \in H_{r}^{1}\left(\mathbb{R}^{N}\right)\left|\int_{\mathbb{R}^{N}} V\right| u\right|^{2} d x<+\infty, \int_{\mathbb{R}^{N}}|\nabla u|^{2}|u|^{2} d x<+\infty\right\}
$$

Set

$$
\|u\|=\left(\int_{\mathbb{R}^{N}}\left(|\nabla u|^{2}+V|u|^{2}\right) d x\right)^{1 / 2} .
$$

A function $u \in X$ is called a weak solution of problem (1.3) if for all $\phi \in C_{0}^{\infty}\left(\mathbb{R}^{N}\right)$ it holds

$$
\int_{\mathbb{R}^{N}}\left(1+u^{2}\right) \nabla u \nabla \phi d x+\int_{\mathbb{R}^{N}}|\nabla u|^{2} u \phi d x+\int_{\mathbb{R}^{N}} V u \phi d x-\lambda \int_{\mathbb{R}^{N}}|u|^{p-2} u \phi d x=0 .
$$

Define the energy functional $I$ on $X$ by

$$
I(u)=\frac{1}{2} \int_{\mathbb{R}^{N}}\left(1+u^{2}\right)|\nabla u|^{2} d x+\frac{1}{2} \int_{\mathbb{R}^{N}} V|u|^{2} d x-\frac{\lambda}{p} \int_{\mathbb{R}^{N}}|u|^{p} d x .
$$

Formally, our problem has a variational structure. Given $u \in X$ and $\phi \in C_{0}^{\infty}\left(\mathbb{R}^{N}\right)$, the Gateaux derivative of $I$ in the direction $\phi$ at $u$, denoted by $\left\langle I^{\prime}(u), \phi\right\rangle$, is defined as $\lim _{t \rightarrow 0^{+}} \frac{I(t u+\phi)-I(u)}{t}$. It is easy to check that

$$
\left\langle I^{\prime}(u), \phi\right\rangle=\int_{\mathbb{R}^{N}}\left[\left(1+u^{2}\right) \nabla u \nabla \phi+|\nabla u|^{2} u \phi+V u \phi-\lambda|u|^{p-2} u \phi\right] d x .
$$


Hence, $u$ is a weak solution of problem (1.3) if this derivative is zero in every direction $\phi \in C_{0}^{\infty}\left(\mathbb{R}^{N}\right)$. In particular, for $u \in X$, we denote

$$
\gamma(u)=\left\langle I^{\prime}(u), u\right\rangle=\int_{\mathbb{R}^{N}}\left(1+2 u^{2}\right)|\nabla u|^{2} d x+\int_{\mathbb{R}^{N}} V|u|^{2} d x-\lambda \int_{\mathbb{R}^{N}}|u|^{p} d x .
$$

Note that we do not claim that $I$ is well-defined nor of class $C^{1}$ in $X$.

From [32], we have the following two lemmas.

Lemma 2.1. For $N \geq 2$, there is a constant $C=C(N)>0$ such that

$$
|u(x)| \leq C|x|^{\frac{1-N}{2}}\|u\|_{H^{1}}
$$

for any $|x| \geq 1$ and $u \in H_{r}^{1}\left(\mathbb{R}^{N}\right)$.

Lemma 2.2. Let $\left\{u_{n}\right\} \subset H_{r}^{1}\left(\mathbb{R}^{N}\right)$ satisfy $u_{n} \rightarrow u$ in $H^{1}\left(\mathbb{R}^{N}\right)$. Then

$$
\liminf _{n} \int_{\mathbb{R}^{N}}\left|\nabla u_{n}\right|^{2}\left|u_{n}\right|^{2} d x \geq \int_{\mathbb{R}^{N}}|\nabla u|^{2}|u|^{2} d x .
$$

The following lemma was first proved by Strauss [36].

Lemma 2.3. Let $N \geq 2$ and $2<q<2^{*}$. Then the imbedding

$$
H_{r}^{1}\left(\mathbb{R}^{N}\right) \hookrightarrow L^{q}\left(\mathbb{R}^{N}\right)
$$

is compact.

Lemma 2.4. (Brézis-Lieb lemma [6]) Let $\left\{u_{n}\right\} \subset L^{q}\left(\mathbb{R}^{N}\right)$ be a bounded sequence, where $1 \leq q<\infty$, such that $u_{n} \rightarrow u$ almost everywhere in $\mathbb{R}^{N}$. Then

$$
\lim _{n \rightarrow \infty}\left(\left|u_{n}\right|_{q}^{q}-\left|u_{n}-u\right|_{q}^{q}\right)=|u|_{q}^{q} .
$$

Lemma 2.5. ([26]) Let $u$ be a weak solution of (1.3). Then $u$ and $\nabla u$ are bounded. Moreover, $u$ satisfies the following exponential decay at infinity

$$
|u(x)| \leq C e^{-\delta R},|x|=R, \int_{\mathbb{R}^{N} \backslash B_{R}}\left(|\nabla u|^{2}+|u|^{2}\right) d x \leq C e^{-\delta R},
$$

for some positive constants $C, \delta$.

Let $\Omega$ be one of the following three types of domains:

$$
\begin{aligned}
& \left\{x \in \mathbb{R}^{N}|| x \mid<R_{1}\right\}, \\
& \left\{x \in \mathbb{R}^{N}\left|0<R_{2} \leq\right| x \mid<R_{3}<+\infty\right\}, \\
& \left\{x \in \mathbb{R}^{N}|| x \mid \geq R_{4}>0\right\} .
\end{aligned}
$$

Set

$$
H_{0, r}^{1}(\Omega)=\left\{u \in H_{0}^{1}(\Omega) \mid u(x)=u(|x|)\right\}
$$

and

$$
X(\Omega)=\left\{\left.u \in H_{0, r}^{1}(\Omega)\left|\int_{\Omega} V u^{2} d x<+\infty, \int_{\Omega}\right| \nabla u\right|^{2} u^{2} d x<+\infty\right\} .
$$


Now we consider the following equation on $\Omega$ :

$$
\left\{\begin{array}{l}
-\Delta u+V u-\frac{1}{2} \Delta\left(|u|^{2}\right) u=\lambda|u|^{p-2} u, \quad x \in \Omega, \\
\left.u\right|_{\partial \Omega}=0
\end{array}\right.
$$

Corresponding to (2.2), we define the functional

$$
I_{\Omega}(u)=\frac{1}{2} \int_{\Omega}\left(1+u^{2}\right)|\nabla u|^{2} d x+\frac{1}{2} \int_{\Omega} V|u|^{2} d x-\frac{\lambda}{p} \int_{\Omega}|u|^{p} d x, u \in X(\Omega) .
$$

Similarly we can define the Gateaux derivative of $I_{\Omega}$ at $u \in X(\Omega)$ and weak solutions of problem (2.2). Set

$$
\gamma_{\Omega}(u)=\left\langle I_{\Omega}^{\prime}(u), u\right\rangle=\int_{\Omega}\left(1+2 u^{2}\right)|\nabla u|^{2} d x+\int_{\Omega} V|u|^{2} d x-\lambda \int_{\Omega}|u|^{p} d x
$$

and define

$$
M(\Omega)=\left\{u \in X(\Omega) \backslash\{0\} \mid \gamma_{\Omega}(u)=0\right\} .
$$

REMARK 2.1. If $\Omega$ is of the second or the third shape of (2.1), then by Lemma 2.1 $X(\Omega)$ is a subspace of $H_{0, r}^{1}(\Omega)$ and $I_{\Omega}$ is well defined and $C^{1}$ smooth in $X(\Omega)$. Hence, in these two cases, we can obtain solutions of problem (2.2) much easier by using the variational formulation.

From [26] we have the following lemma.

Lemma 2.6. Suppose that $p>4$ and $u \in X(\Omega)$. Then there is a unique $t>0$ such that $t u \in M(\Omega)$. Moreover, if $\gamma_{\Omega}(u)<0$, then $t \in(0,1)$.

Lemma 2.7. Suppose that the domain $\Omega$ is one of the forms of (2.1). Then $c_{1}=$ $\inf _{M(\Omega)} I_{\Omega}(u)$ can be achieved by some positive function $u$ which is a solution of problem $(2.2)$, i.e., $\forall \phi \in C_{0}^{\infty}(\Omega)$ it holds

$$
\int_{\Omega}\left[\left(1+u^{2}\right) \nabla u \nabla \phi+|\nabla u|^{2} u \phi+V u \phi-\lambda|u|^{p-2} u \phi\right] d x=0 .
$$

Moreover, the above equation holds for $\phi \in X(\Omega)$ with the property that $\int_{\Omega} u^{2}|\nabla \phi|^{2} d x<\infty$ and $\int_{\Omega}|\nabla u|^{2} \phi^{2} d x<\infty$.

Proof. Since the quasilinear operator $\Delta\left(.^{2}\right)$. appears, we can not use the Mountain-Pass theorem here. Now we use a minimization method. The proof can be divided into three steps.

Step 1. $c_{1}$ is attained.

By the definition of $c_{1}$, there exists a sequence $\left\{u_{n}\right\} \subset M(\Omega)$ such that

$$
I_{\Omega}\left(u_{n}\right)=c_{1}+o(1)
$$

i.e.,

$$
\begin{aligned}
& 0=\int_{\Omega}\left(\left|\nabla u_{n}\right|^{2}+V\left|u_{n}\right|^{2}\right) d x+2 \int_{\Omega} u_{n}^{2}\left|\nabla u_{n}\right|^{2} d x-\lambda \int_{\Omega}\left|u_{n}\right|^{p} d x \\
& c_{1}+o(1)=I_{\Omega}\left(u_{n}\right)=\frac{1}{2} \int_{\Omega}\left(\left|\nabla u_{n}\right|^{2}+V\left|u_{n}\right|^{2}\right) d x+\frac{1}{2} \int_{\Omega} u_{n}^{2}\left|\nabla u_{n}\right|^{2} d x-\frac{\lambda}{p} \int_{\Omega}\left|u_{n}\right|^{p} d x .
\end{aligned}
$$


Thus we have

$$
c_{1}+o(1)=\left(\frac{1}{2}-\frac{1}{p}\right)\left\|u_{n}\right\|^{2}+\left(\frac{1}{2}-\frac{2}{p}\right) \int_{\Omega} u_{n}^{2}\left|\nabla u_{n}\right|^{2} d x .
$$

From $p>4$, it is easy to verify that $\left\{u_{n}\right\}$ is bounded in $X(\Omega)$. Hence, by Lemma 2.3, we can extract a subsequence of $\left\{u_{n}\right\}$ (still denoted by $\left\{u_{n}\right\}$ ), such that

$$
\begin{array}{ll}
u_{n} \rightarrow u & \text { in } \quad X(\Omega), \\
u_{n} \rightarrow u & \text { in } \quad L^{q}(\Omega), 2<q<2^{*} .
\end{array}
$$

Since $\nabla\left(u_{n}^{2}\right)$ is uniformly bounded in $L^{2}(\Omega)$ from (2.4), by Sobolev's inequality we have $\left|u_{n}^{2}\right|_{2^{*}} \leq C$, which gives $\left|u_{n}\right|_{22^{*}} \leq C$. By Hölder's inequality we have

$$
u_{n} \rightarrow u \quad \text { in } \quad L^{p}(\Omega), 2<p<22^{*} .
$$

Next we want to prove $u_{n} \rightarrow u$ in $X(\Omega)$. Let $\nu_{n}=u_{n}-u, u_{n} \in M(\Omega)$. Then by Lemmas $2.2,2.4$ and 2.5 ,

$$
\begin{aligned}
0=\lim _{n \rightarrow \infty} \gamma_{\Omega}\left(u_{n}\right) & =\lim _{n \rightarrow \infty}\left\|u_{n}\right\|^{2}+2\left|u_{n} \nabla u_{n}\right|_{2}^{2}-\lambda\left|u_{n}\right|_{p}^{p} \\
& \geq \lim _{n \rightarrow \infty}\left\|\nu_{n}\right\|^{2}+\|u\|^{2}+2|u \nabla u|_{2}^{2}-\lambda|u|_{p}^{p} \\
& =\gamma_{\Omega}(u)+\lim _{n \rightarrow \infty}\left\|\nu_{n}\right\|^{2},
\end{aligned}
$$

so that $\gamma_{\Omega}(u) \leq 0$. If $\gamma_{\Omega}(u)<0$, then by Lemma 2.6 there exists $t \in(0,1)$ such that $t u \in M(\Omega)$. From $\gamma_{\Omega}\left(u_{n}\right)=0$, we have

$$
2\left|u_{n} \nabla u_{n}\right|_{2}^{2}=-\left\|u_{n}\right\|^{2}+\lambda\left|u_{n}\right|_{p}^{p}
$$

so that

$$
I_{\Omega}\left(u_{n}\right)=\frac{1}{4}\left\|u_{n}\right\|^{2}+\left(\frac{1}{4}-\frac{1}{p}\right) \lambda\left|u_{n}\right|_{p}^{p}
$$

Therefore we get

$$
\begin{aligned}
c_{1}=\lim _{n \rightarrow \infty} I_{\Omega}\left(u_{n}\right) & =\frac{1}{4}\|u\|^{2}+\left(\frac{1}{4}-\frac{1}{p}\right) \lambda|u|_{p}^{p}+\frac{1}{4} \lim _{n \rightarrow \infty}\left\|\nu_{n}\right\|^{2} \\
& \geq \frac{1}{4}\|u\|^{2}+\left(\frac{1}{4}-\frac{1}{p}\right) \lambda|u|_{p}^{p} \\
& =\frac{1}{4} t^{-2}\|t u\|^{2}+\left(\frac{1}{4}-\frac{1}{p}\right) \lambda t^{-p}|t u|_{p}^{p} .
\end{aligned}
$$

For $t \in(0,1)$, we have

$$
c_{1}>\frac{1}{4}\|t u\|^{2}+\left(\frac{1}{4}-\frac{1}{p}\right) \lambda|t u|_{p}^{p}=I_{\Omega}(t u)
$$

which contradicts the definition of $c_{1}$. Thus we get

$$
\gamma_{\Omega}(u)=0 \text { and } u \in M(\Omega) .
$$


Combining (2.6) and (2.8), we obtain

$$
\lim _{n \rightarrow \infty}\left\|\nu_{n}\right\|^{2}=0 .
$$

Substituting (2.8) and (2.9) into (2.7), we see

$$
I_{\Omega}(u)=c_{1},
$$

and $c_{1}$ is attained by $u$.

Step 2. $u$ is a radial solution of (2.2).

We use an indirect argument which is similar to [26]. Suppose that $u \in$ $M(\Omega), I_{\Omega}(u)=c_{1}$, but the conclusion (2.3) of the lemma is not true. Then first we can find a function $\phi \in X(\Omega)$ with the property that

$$
\int_{\Omega} u^{2}|\nabla \phi|^{2} d x<\infty \quad \text { and } \quad \int_{\Omega}|\nabla u|^{2} \phi^{2} d x<\infty
$$

but

$$
\left\langle I_{\Omega}^{\prime}(u), \phi\right\rangle=\int_{\Omega}\left[\left(1+u^{2}\right) \nabla u \nabla \phi+|\nabla u|^{2} u \phi+V u \phi-\lambda|u|^{p-2} u \phi\right] d x \leq-1 .
$$

Choose $\varepsilon>0$ small enough such that

$$
\left\langle I_{\Omega}^{\prime}(t u+\sigma \phi), \phi\right\rangle \leq-\frac{1}{2}, \quad \forall|t-1|+|\sigma| \leq \varepsilon .
$$

Let $\eta$ be a cut-off function such that

$$
\eta(t)= \begin{cases}1, & |t-1| \leq \frac{1}{2} \varepsilon \\ 0, & |t-1| \geq \varepsilon\end{cases}
$$

We estimate $\sup _{t} I_{\Omega}(t u+\varepsilon \eta(t) \phi)$. If $|t-1| \leq \varepsilon$, then

$$
\begin{aligned}
I_{\Omega}(t u+\varepsilon \eta(t) \phi) & =I_{\Omega}(t u)+\int_{0}^{1}\left\langle I_{\Omega}^{\prime}(t u+\sigma \varepsilon \eta(t) \phi), \varepsilon \eta(t) \phi\right\rangle d \sigma \\
& \leq I_{\Omega}(t u)-\frac{1}{2} \varepsilon \eta(t) .
\end{aligned}
$$

If $|t-1| \geq \varepsilon$, then $\eta(t)=0$, and the above estimate is trivial. Now since $u \in M(\Omega)$, for $t \neq 1$ we get $I_{\Omega}(t u)<I_{\Omega}(u)$. Hence it follows from (2.10) that

$$
I_{\Omega}(t u+\varepsilon \eta(t) \phi) \leq \begin{cases}I_{\Omega}(t u)<I_{\Omega}(u), & \text { if } t \neq 1, \\ I_{\Omega}(u)-\frac{1}{2} \varepsilon \eta(1)=I_{\Omega}(u)-\frac{1}{2} \varepsilon, & \text { if } t=1 .\end{cases}
$$

In any case we have $I_{\Omega}(t u+\varepsilon \eta(t) \phi)<I_{\Omega}(u)=c_{1}$. In particular,

$$
\sup _{0 \leq t \leq 2} I_{\Omega}(t u+\varepsilon \eta(t) \phi)<c_{1} .
$$

Since $u \in M(\Omega)$, we have

$$
\int_{\Omega}\left[\left(|\nabla u|^{2}+V u^{2}\right)+2 u^{2}|\nabla u|^{2}-\lambda|u|^{p}\right] d x=0 .
$$


Let

$$
\begin{aligned}
h(t)=\int_{\Omega} & {\left[|\nabla(t u+\varepsilon \eta(t) \phi)|^{2}+V|t u+\varepsilon \eta(t) \phi|^{2}+2|t u+\varepsilon \eta(t) \phi|^{2}|\nabla(t u+\varepsilon \eta(t) \phi)|^{2}\right.} \\
& \left.-\lambda|t u+\varepsilon \eta(t) \phi|^{p}\right] d x .
\end{aligned}
$$

Without loss of generality, we assume $\varepsilon<\frac{1}{4}$. For $t=2$, we have $\eta(2)=0$, and thus from (2.12),

$$
\begin{aligned}
h(2) & =\int_{\Omega}\left[4\left(|\nabla u|^{2}+V|u|^{2}\right)+32|u|^{2}|\nabla u|^{2}-2^{p} \lambda|u|^{p}\right] d x \\
& =\left(4-2^{p}\right) \int_{\Omega}\left(|\nabla u|^{2}+V|u|^{2}\right) d x+\left(32-2^{p+1}\right) \int_{\Omega}|u|^{2}|\nabla u|^{2} d x \\
& <0 .
\end{aligned}
$$

For $t=\frac{1}{2}$, we see

$$
\begin{aligned}
h\left(\frac{1}{2}\right) & =\int_{\Omega}\left[\frac{1}{4}\left(|\nabla u|^{2}+V|u|^{2}\right)+\frac{1}{8}|u|^{2}|\nabla u|^{2}-\frac{1}{2^{p}} \lambda|u|^{p}\right] d x \\
& =\left(\frac{1}{4}-\frac{1}{2^{p}}\right) \int_{\Omega}\left(|\nabla u|^{2}+V|u|^{2}\right) d x+\left(\frac{1}{8}-\frac{1}{2^{p-1}}\right) \int_{\Omega}|u|^{2}|\nabla u|^{2} d x \\
& >0 .
\end{aligned}
$$

As a result, we can find $\bar{t} \in\left(\frac{1}{2}, 2\right)$ such that $h(\bar{t})=0$, which implies that $\bar{t} u+\varepsilon \eta(\bar{t}) \phi \in$ $M(\Omega)$. However, it follows from $(2.11)$ that $I_{\Omega}(\bar{t} u+\varepsilon \eta(\bar{t}) \phi)<c_{1}$. Hence, we get a contradiction.

Step 3. $u>0$.

Firstly, by Lemma 2.5 , we have $u,|\nabla u| \in L^{\infty}(\Omega)$. Moreover, by the condition $V(x) \in C\left(\mathbb{R}^{N}, \mathbb{R}\right)$ and the $L^{p}$ estimate, we know that $u \in W_{\text {loc }}^{2, p}(\Omega)$ for any $p<+\infty$. Hence $u \in C_{l o c}^{1, \alpha}(\Omega), \alpha \in(0,1)$. Since $u$ satisfies the equation

$$
-u_{r r}-\frac{N-1}{r} u_{r}=\frac{\lambda|u|^{p-2}-V+\left|u_{r}\right|^{2}}{1+u^{2}} u
$$

we know that $u_{r r}$ is continuous, except possibly at 0 . Set $G(r)=\frac{\lambda|u|^{p-2}-V+\left|u_{r}\right|^{2}}{1+u^{2}} u$, and note that $G(r)$ is continuous on $[0,+\infty)$. Rewriting $(2.13)$ as $-\frac{d}{d r}\left(r^{N-1} u_{r}\right)=r^{N-1} G(r)$ and integrating from 0 to $r$, we find

$$
r^{N-1} u_{r}=-\int_{0}^{r} s^{N-1} G(s) d s .
$$

With a change of variable, we get

$$
r^{N-1} u_{r}=-r \int_{0}^{1} s^{N-1} G(s r) d s \quad \text { or } \quad \frac{u_{r}}{r}=-\int_{0}^{1} s^{N-1} G(s r) d s .
$$

Since

$$
\int_{0}^{1} s^{N-1} G(s r) d s \rightarrow \frac{G(0)}{N} \quad \text { as } \quad r \rightarrow 0
$$


we deduce that $u_{r r}(0)$ exists and $u_{r r}(0)=\frac{G(0)}{N}$. Furthermore, from Equation (2.13) we see that

$$
u_{r r} \rightarrow-\frac{G(0)}{N} \quad \text { as } \quad r \rightarrow 0,
$$

and thus $u \in C^{2}(\Omega)$.

Secondly, we try to verify that the minimizer of $c_{1}$ will not change sign. If the attained function $u$ changes sign in $\Omega$, then $u^{+}, u^{-} \in M(\Omega)$, where $u^{+}=\max \{u, 0\}, u^{-}=$ $-\min \{u, 0\}$. Thus

$$
I_{\Omega}\left(u^{+}\right)<I_{\Omega}(u)=\inf _{M(\Omega)} I_{\Omega}(u) \leq I_{\Omega}\left(u^{+}\right),
$$

which is a contradiction. Therefore either $u \geq 0$ or $u \leq 0$. Without loss of generality, we can assume $u \geq 0$. Now we show that $u>0$. If there exists $x_{0}$ such that $u\left(x_{0}\right)=0$, then $u^{\prime}\left(x_{0}\right)=0$ for $u \geq 0$. By the Strong Maximum Principle (e.g., Gilbarg and Trudinger [18]), $u=0$ near $x_{0}$ and $u$ will vanish identically, which is impossible since $u \in M(\Omega)$. Hence $u>0$ and we complete the proof.

\section{The proof of Theorem 1.1}

In this section we will consider the existence of the nodal solutions of (1.3). For any given $k+2$ numbers $r_{j}(j=0,1, \cdots, k+1)$ such that $0=r_{0}<r_{1}<r_{2}<\cdots<r_{k}<$ $r_{k+1}=+\infty$, denote

$$
\begin{aligned}
& \Omega^{1}=\left\{x \in \mathbb{R}^{N}:|x|<r_{1}\right\}, \\
& \Omega^{j}=\left\{x \in \mathbb{R}^{N}: r_{j-1}<|x|<r_{j}\right\} .
\end{aligned}
$$

We will always extend $u_{j} \in X\left(\Omega^{j}\right)$ to $X$ by setting $u \equiv 0$ on $x \in \mathbb{R}^{N} \backslash \Omega^{j}$ for every $u_{j} \in X\left(\Omega^{j}\right), j=1,2, \cdots, k+1$. In this sense, we use $I\left(u_{j}\right)$ to replace $I_{\Omega^{j}}\left(u_{j}\right)$ and $\gamma\left(u_{j}\right)$ to replace $\gamma_{\Omega^{j}}\left(u_{j}\right)$ in the sequel.

Define

$$
\begin{gathered}
Y_{k}^{ \pm}\left(r_{1}, r_{2}, \cdots, r_{k+1}\right)=\left\{u \in X \mid u= \pm \sum_{j=1}^{k+1}(-1)^{j-1} u_{j}, u_{j} \geq 0,\right. \\
\left.u_{j} \not \equiv 0, u_{j} \in X\left(\Omega^{j}\right), j=1,2, \cdots, k+1\right\}, \\
M_{k}^{ \pm}=\left\{u \in X \mid \exists 0<r_{1}<r_{2}<\cdots<r_{k}<r_{k+1}=+\infty,\right. \\
\left.\quad \text { such that } u \in Y_{k}^{ \pm}\left(r_{1}, r_{2}, \cdots, r_{k+1}\right) \text { and } u_{j} \in M\left(\Omega^{j}\right), j=1,2, \cdots, k+1\right\} .
\end{gathered}
$$

Note that $M_{k}^{ \pm} \neq \emptyset, k=1,2, \cdots$. In the following we will always refer to $M_{k}$ and we will drop the "+". For $M_{k}^{-}$, everything could be done exactly in the same way. By the arguments of the standard Nehari method [29], it is easy to verify that

$$
\forall u=\sum_{j=1}^{k+1}(-1)^{j-1} u_{j} \in M_{k} \Longleftrightarrow I(u)=\max _{\substack{\alpha_{j}>0 \\ 1 \leq j \leq k+1}} I\left(\sum_{j=1}^{k+1} \alpha_{j} \tilde{u}_{j}\right),
$$

where $\tilde{u}_{j}=(-1)^{j-1} u_{j}$.

Set

$$
c_{k}=\inf _{M_{k}} I(u), k=1,2, \cdots
$$


LeMma 3.1. $c_{k}$ is attained provided that $4<p<22^{*}, k=0,1, \cdots$.

Proof. We will prove by induction that for each $k$ there exists $u_{k} \in M_{k}$ such that

$$
I\left(u_{k}\right)=c_{k} .
$$

The case that $k=0$ can be deduced by setting $\Omega=\mathbb{R}^{N}$ in Lemma 2.7. We discuss the case $k \geq 1$ in the following.

Firstly, we prove $I$ is bounded from below on $M_{k}$ by a positive constant. Let $u \in M_{k}$; then $u=\sum_{j=1}^{k+1}(-1)^{j-1} u_{j}$ and $u_{j} \in M\left(\Omega^{j}\right), j=1,2, \cdots, k+1$. Denote

$$
\eta_{j}^{2}=\int_{\Omega^{j}}\left(1+u_{j}^{2}\right)\left|\nabla u_{j}\right|^{2} d x+\int_{\Omega^{j}} V\left|u_{j}\right|^{2} d x .
$$

By Hölder's inequality and Sobolev's inequality we have, with $\theta=(p-2)(N-$ $2) / 2(N+2)$,

$$
\begin{aligned}
\int_{\Omega^{j}}\left|u_{j}\right|^{p} d x & \leq\left(\int_{\Omega^{j}}\left|u_{j}\right|^{2} d x\right)^{1-\theta}\left(\int_{\Omega^{j}}\left|u_{j}\right|^{\frac{4 N}{N-2}} d x\right)^{\theta} \\
& \leq C\left(\int_{\Omega^{j}}\left|u_{j}\right|^{2} d x\right)^{1-\theta}\left(\int_{\Omega^{j}} u_{j}^{2}\left|\nabla u_{j}\right|^{2} d x\right)^{\frac{\theta N}{N-2}} \\
& \leq C \eta_{j}^{2(1-\theta)} \eta_{j}^{\frac{2 \theta N}{N-2}} \\
& =C \eta_{j}^{2+\frac{2(p-2)}{N+2}}
\end{aligned}
$$

Then

$$
\begin{aligned}
0 & =\int_{\Omega^{j}}\left(1+2 u_{j}^{2}\right)\left|\nabla u_{j}\right|^{2} d x+\int_{\Omega^{j}} V\left|u_{j}\right|^{2} d x-\int_{\Omega^{j}}\left|u_{j}\right|^{p} d x \\
& \geq \eta_{j}^{2}-C \eta_{j}^{2+\frac{2(p-2)}{N+2}}
\end{aligned}
$$

from which

$$
\eta_{j}^{2} \geq C_{j}>0
$$

Then from (3.2) and $p>4$,

$$
\begin{aligned}
I(u) & =I\left(\sum_{j=1}^{k+1}(-1)^{j-1} u_{j}\right)=\sum_{j=1}^{k+1} I\left(u_{j}\right) \\
& =\sum_{j=1}^{k+1}\left\{\frac{1}{2} \int_{\Omega^{j}}\left(1+u_{j}^{2}\right)\left|\nabla u_{j}\right|^{2} d x+\frac{1}{2} \int_{\Omega^{j}} V\left|u_{j}\right|^{2} d x-\frac{\lambda}{p} \int_{\Omega^{j}}\left|u_{j}\right|^{p} d x\right\} \\
& =\sum_{j=1}^{k+1}\left\{\left(\frac{1}{2}-\frac{1}{p}\right) \int_{\Omega^{j}}\left(\left|\nabla u_{j}\right|^{2}+V\left|u_{j}\right|^{2}\right) d x+\left(\frac{1}{2}-\frac{2}{p}\right) \int_{\Omega^{j}}\left|\nabla u_{j}\right|^{2}\left|u_{j}\right|^{2} d x\right\} \\
& \geq\left(\frac{1}{2}-\frac{2}{p}\right) \sum_{j=1}^{k+1} C_{j}>0 .
\end{aligned}
$$


Hence $I$ is bounded from below on $M_{k}$ by a positive constant.

Secondly, we suppose the claim is true for $k-1$ and let $\left\{u_{m}\right\}_{m \geq 1}$ be a minimizing sequence of $c_{k}$ in $M_{k}$, that is,

$$
\lim _{m \rightarrow \infty} I\left(u_{m}\right)=c_{k}, \quad u_{m} \in M_{k}, \quad m=1,2, \cdots .
$$

$u_{m}$ corresponds to $k$ nodes, $r_{m}^{1}, r_{m}^{2}, \cdots, r_{m}^{k}$, with $0<r_{m}^{1}<r_{m}^{2}<\cdots<r_{m}^{k}<+\infty$. Set

$$
\Omega_{m}^{i}=\left\{x \in \mathbb{R}^{N}\left|r_{m}^{i-1}<\right| x \mid<r_{m}^{i}\right\},
$$

and

$$
u_{m}^{i}= \begin{cases}u_{m}, & x \in \Omega_{m}^{i}, \\ 0, & x \notin \Omega_{m}^{i}\end{cases}
$$

By selecting a subsequence, we may assume that $\lim _{m \rightarrow \infty} r_{m}^{i}=r^{i}$, and clearly $0 \leq r^{1} \leq$ $r^{2} \leq \ldots \leq r^{k} \leq+\infty$. Now we divide the rest of the proof into three steps.

Step 1. $r^{i} \neq r^{i-1}, i=1,2, \cdots, k$. Here we denote $r^{0}=0$.

If there exists some $i \in\{1,2, \cdots, k\}$ such that $r^{i}=r^{i-1}$, then $\lim _{m \rightarrow \infty} r_{m}^{i}=$ $\lim _{m \rightarrow \infty} r_{m}^{i-1}$. We denote the measure of $\Omega_{m}^{i}$ by $\left|\Omega_{m}^{i}\right|$, so that $\left|\Omega_{m}^{i}\right| \rightarrow 0$ as $m \rightarrow \infty$. Since $u_{m}^{i} \in M\left(\Omega_{m}^{i}\right)$,

$$
\begin{aligned}
I\left(u_{m}^{i}\right) & =\frac{1}{2} \int_{\Omega_{m}^{i}}\left(\left|\nabla\left(u_{m}^{i}\right)\right|^{2}+V\left|u_{m}^{i}\right|^{2}\right) d x+\frac{1}{2} \int_{\Omega_{m}^{i}}\left|\nabla u_{m}^{i}\right|^{2}\left|u_{m}^{i}\right|^{2} d x-\frac{\lambda}{p} \int_{\Omega_{m}^{i}}\left|u_{m}^{i}\right|^{p} d x \\
& =\left(\frac{1}{2}-\frac{1}{p}\right) \int_{\Omega_{m}^{i}}\left(\left|\nabla\left(u_{m}^{i}\right)\right|^{2}+V\left|u_{m}^{i}\right|^{2}\right) d x+\left(\frac{1}{2}-\frac{2}{p}\right) \int_{\Omega_{m}^{i}}\left|\nabla u_{m}^{i}\right|^{2}\left|u_{m}^{i}\right|^{2} d x \\
& \geq C \eta^{2}\left(u_{m}^{i}\right)
\end{aligned}
$$

where

$$
\eta^{2}\left(u_{m}^{i}\right)=\int_{\Omega_{m}^{i}}\left(1+\left(u_{m}^{i}\right)^{2}\right)\left|\nabla\left(u_{m}^{i}\right)\right|^{2} d x+\int_{\Omega_{m}^{i}} V\left|u_{m}^{i}\right|^{2} d x .
$$

On the other hand, it follows from Hölder's inequality, Sobolev's inequality, and the fact $u_{m}^{i} \in M\left(\Omega_{m}^{i}\right)$ that

$$
\begin{aligned}
\eta^{2}\left(u_{m}^{i}\right) & \leq \int_{\Omega_{m}^{i}}\left(\left|\nabla\left(u_{m}^{i}\right)\right|^{2}+V\left|u_{m}^{i}\right|^{2}\right) d x+2 \int_{\Omega_{m}^{i}}\left|\nabla u_{m}^{i}\right|^{2}\left|u_{m}^{i}\right|^{2} d x \\
& =\lambda \int_{\Omega_{m}^{i}}\left|u_{m}^{i}\right|^{p} d x \\
& \leq \lambda\left(\int_{\Omega_{m}^{i}}\left|u_{m}^{i}\right|^{22^{*}} d x\right)^{\frac{p}{22^{*}}}\left|\Omega_{m}^{i}\right|^{1-\frac{p}{22^{*}}} \\
& \leq C\left(\int_{\Omega_{m}^{i}}\left|\nabla u_{m}^{i}\right|^{2}\left|u_{m}^{i}\right|^{2} d x\right)^{\frac{p}{4}}\left|\Omega_{m}^{i}\right|^{1-\frac{p}{22^{*}}} \\
& \leq C\left(\eta^{2}\left(u_{m}^{i}\right)\right)^{\frac{p}{4}}\left|\Omega_{m}^{i}\right|^{1-\frac{p}{22^{*}}} .
\end{aligned}
$$


Thus

$$
\left(\eta^{2}\left(u_{m}^{i}\right)\right)^{\frac{p}{4}-1} \geq C\left|\Omega_{m}^{i}\right|^{-1+\frac{p}{22^{*}}} .
$$

Since $4<p<22^{*}$, we have from (3.4) that

$$
\eta^{2}\left(u_{m}^{i}\right) \rightarrow \infty, \quad \text { as } \quad m \rightarrow \infty
$$

So (3.3) implies

$$
I\left(u_{m}^{i}\right) \rightarrow \infty, \quad \text { as } \quad m \rightarrow \infty .
$$

By the inductive assumption and (3.5), for $\varepsilon>0$ fixed we can choose $M>0$ such that

$$
I\left(u_{m}^{i}\right)>c_{k}-c_{k-1}+\varepsilon, \quad\left|I\left(u_{m}\right)-c_{k}\right|<\varepsilon, \quad \text { as } m \geq M .
$$

Then we may define $\hat{u}(x) \in M_{k-1}$ by

$$
\hat{u}(x)= \begin{cases}u_{m}^{l}(x), & x \in \Omega_{m}^{l} \text { as } l<i, \\ 0, & x \in \Omega_{m}^{i}, \\ -u_{m}^{l}(x), & x \in \Omega_{m}^{l} \text { as } l>i .\end{cases}
$$

Hence

$$
I(\hat{u})=I\left(u_{m}\right)-I\left(u_{m}^{i}\right)<c_{k}+\varepsilon-\left(c_{k}-c_{k-1}+\varepsilon\right)=c_{k-1}, \quad \text { as } m \geq M,
$$

which contradicts the fact that $c_{k-1}=\inf _{M_{k-1}} I(u)$. Thus $r^{i} \neq r^{i-1}, i=1,2, \cdots, k$.

Step 2. $r^{k}<+\infty$.

If $r^{k}=+\infty$, then $\lim _{m \rightarrow \infty} r_{m}^{k}=+\infty$. It follows from Lemma 2.1 and $u_{m}^{k} \in M\left(u_{m}^{k}\right)$ that

$$
\begin{aligned}
\eta^{2}\left(u_{m}^{k}\right) & \leq \int_{\Omega_{m}^{k}}\left(\left|\nabla\left(u_{m}^{k}\right)\right|^{2}+V\left|u_{m}^{k}\right|^{2}\right) d x+2 \int_{\Omega_{m}^{k}}\left|\nabla u_{m}^{k}\right|^{2}\left|u_{m}^{k}\right|^{2} d x \\
& =\lambda \int_{\Omega_{m}^{k}}\left|u_{m}^{k}\right|^{p} d x \\
& \leq \int_{\Omega_{m}^{k}}\left|u_{m}^{k}\right|^{2}\left|u_{m}^{k}\right|^{p-2} d x \\
& \leq\left\|u_{m}^{k}\right\|^{p-2} \int_{\Omega_{m}^{k}}\left|u_{m}^{k}\right|^{2}|x|^{\frac{(1-N)(p-2)}{2}} d x \\
& \leq C\left(\eta^{2}\left(u_{m}^{k}\right)\right)^{\frac{p}{2}}\left|r_{m}^{k}\right|^{\frac{(1-N)(p-2)}{2}} .
\end{aligned}
$$

Thus

$$
\eta^{2}\left(u_{m}^{k}\right) \geq C\left|r_{m}^{k}\right|^{N-1}
$$

From (3.6) we have

$$
\eta^{2}\left(u_{m}^{k}\right) \rightarrow \infty, \quad \text { as } \quad m \rightarrow \infty .
$$


So by (3.3) we find

$$
I\left(u_{m}^{k}\right) \rightarrow \infty, \quad \text { as } \quad m \rightarrow \infty .
$$

Repeating Step 1, we can obtain $r^{k}<+\infty$.

Step 3. $c_{k}$ is attained.

Using an argument similar to that in the proof of Lemma 2.7, we can find a subsequence (still denoted by $\left\{u_{m}\right\}$ ) such that

$$
\begin{array}{ll}
u_{m} \rightarrow u & \text { in } X, \\
u_{m} \rightarrow u & \text { in } L^{p}\left(\mathbb{R}^{N}\right) .
\end{array}
$$

Set $\Omega^{i}=\left\{x \in \mathbb{R}^{N}\left|r^{i-1}<\right| x \mid<r^{i}\right\}$, for all $i=1,2, \cdots, k+1, r^{0}=0$, and $r^{k+1}=+\infty$. Lemma 2.7 implies that $\bar{c}=\inf _{M\left(\Omega^{i}\right)} I(u)$ is attained by some positive function $\hat{u}^{i}$ which satisfies the following boundary value problem

$$
\left\{\begin{array}{l}
-\Delta u+V u-\frac{1}{2} \Delta\left(|u|^{2}\right) u=\lambda|u|^{p-2} u, \quad x \in \Omega^{i}, \\
\left.u\right|_{\partial \Omega^{i}}=0 .
\end{array}\right.
$$

Define $u_{k}=\sum_{i=1}^{k+1}(-1)^{i-1} \hat{u}^{i}(x),\left(\hat{u}^{i}(x)=0, x \notin \Omega^{i}\right)$. Then, clearly, $u_{k} \in M_{k}$. Consider the coordinate transformations

by
\[ \Phi_{m}(x)=\varphi_{m}(|x|) \frac{x}{|x|}, \quad x \in \mathbb{R}^{N}, \]
where $\varphi_{m}(r)=\frac{\left(r^{i}-r^{i-1}\right)\left(r-r_{m}^{i-1}\right)}{r_{m}^{i}-r_{m}^{i-1}}+r^{i-1}$. For any $r \in \mathbb{R}$, clearly, $\Phi_{m}\left(\Omega_{m}^{i}\right)=\Omega^{i}$.

$$
\Phi_{m}: \mathbb{R}^{N} \longrightarrow \mathbb{R}^{N}, \quad m=1,2, \cdots,
$$

Let $y=\Phi_{m}(x) \in \Omega^{i}$, if $x \in \Omega_{m}^{i}$. It is easy to show that

$$
\begin{gathered}
|\nabla u(y)|=\left(R_{m}^{i}\right)^{-1}|\nabla u(x)|, \\
d y=\left|J_{m}^{i}\right| d x,
\end{gathered}
$$

and

$$
a_{m}^{i} \leq\left(\frac{\Phi_{m}(r)}{r}\right)^{N-1} \leq A_{m}^{i}
$$

where

$$
\begin{gathered}
R_{m}^{i}=\frac{r^{i}-r^{i-1}}{r_{m}^{i}-r_{m}^{i-1}}, \quad J_{m}^{i}=\left[\varphi_{m}(|x|)\right]^{N-1}\left(\varphi_{m}(|x|)\right)^{\prime}|x|^{1-N} \\
a_{m}^{i}=\left(\min \left\{r^{i} / r_{m}^{i}, r^{i-1} / r_{m}^{i-1}\right\}\right)^{N-1}
\end{gathered}
$$


and

Clearly,

$$
A_{m}^{i}=\left(\max \left\{r^{i} / r_{m}^{i}, r^{i-1} / r_{m}^{i-1}\right\}\right)^{N-1} .
$$

$$
a_{m}^{i} R_{m}^{i} \leq\left|J_{m}^{i}\right| \leq A_{m}^{i} R_{m}^{i}
$$

and

$$
R_{m}^{i} \rightarrow 1, \quad a_{m}^{i} \rightarrow 1, \quad A_{m}^{i} \rightarrow 1, \quad J_{m}^{i} \rightarrow 1, \quad \text { as } m \rightarrow \infty .
$$

Let

$$
f(t)=t^{2} \int_{\Omega^{i}}\left(\left|\nabla\left(u_{m}^{i}\right)\right|^{2}+V\left|u_{m}^{i}\right|^{2}\right) d y+2 t^{4} \int_{\Omega^{i}}\left|\nabla u_{m}^{i}\right|^{2}\left|u_{m}^{i}\right|^{2} d y-\lambda t^{p} \int_{\Omega^{i}}\left|u_{m}^{i}\right|^{p} d y .
$$

Since $p>4$, there exist some $t_{m}^{i}>0$, such that $f\left(t_{m}^{i}\right)=0$, thus $t_{m}^{i} u_{m}^{i} \in M\left(\Omega^{i}\right)$.

Now, we claim that

$$
t_{m}^{i} \rightarrow 1 \quad \text { as } m \rightarrow \infty, \quad i=1,2, \cdots, k .
$$

Indeed, since $f\left(t_{m}^{i}\right)=0$, we have

$$
\begin{aligned}
\int_{\Omega^{i}}\left(\left|\nabla\left(u_{m}^{i}\right)\right|^{2}+V(y)\left|u_{m}^{i}\right|^{2}\right) d y & +2\left(t_{m}^{i}\right)^{2} \int_{\Omega^{i}}\left|\nabla u_{m}^{i}\right|^{2}\left|u_{m}^{i}\right|^{2} d y \\
& -\lambda\left(t_{m}^{i}\right)^{p-2} \int_{\Omega^{i}}\left|u_{m}^{i}\right|^{p} d y=0 .
\end{aligned}
$$

We can prove that there exists a constant $M>0$ such that

$$
0<t_{m}^{i} \leq M<\infty .
$$

By selecting a subsequence, we may assume that $\lim _{m \rightarrow \infty} t_{m}^{i}=t_{*}^{i}$. Using (3.8)-(3.12), we have that

$$
\begin{gathered}
\lim _{m \rightarrow \infty} \int_{\Omega^{i}}\left|\nabla u_{m}^{i}(y)\right|^{2} d y=\lim _{m \rightarrow \infty} \int_{\Omega_{m}^{i}}\left|\nabla u_{m}^{i}(x)\right|^{2} d x \\
\lim _{m \rightarrow \infty} \int_{\Omega^{i}} V(y)\left|u_{m}^{i}(y)\right|^{2} d y=\lim _{m \rightarrow \infty} \int_{\Omega_{m}^{i}} V(x)\left|u_{m}^{i}(x)\right|^{2} d x, \\
\lim _{m \rightarrow \infty} \int_{\Omega^{i}}\left|\nabla u_{m}^{i}(y)\right|^{2}\left|u_{m}^{i}(y)\right|^{2} d y=\lim _{m \rightarrow \infty} \int_{\Omega_{m}^{i}}\left|\nabla u_{m}^{i}(x)\right|^{2}\left|u_{m}^{i}(x)\right|^{2} d x, \\
\lim _{m \rightarrow \infty} \int_{\Omega^{i}}\left|u_{m}^{i}\right|^{p} d y=\lim _{m \rightarrow \infty} \int_{\Omega_{m}^{i}}\left|u_{m}^{i}\right|^{p} d x .
\end{gathered}
$$

Substituting (3.15)-(3.18) into (3.14), we find that

$$
\begin{aligned}
\lim _{m \rightarrow \infty}\left[\int_{\Omega_{m}^{i}}\left(\left|\nabla\left(u_{m}^{i}\right)\right|^{2}+V\left|u_{m}^{i}\right|^{2}\right) d x\right. & +2\left(t_{*}^{i}\right)^{2} \int_{\Omega_{m}^{i}}\left|\nabla u_{m}^{i}\right|^{2}\left|u_{m}^{i}\right|^{2} d x \\
& \left.-\lambda\left(t_{*}^{i}\right)^{p-2} \int_{\Omega_{m}^{i}}\left|u_{m}^{i}\right|^{p} d x\right]=0 .
\end{aligned}
$$


Let

$$
\begin{aligned}
& \lim _{m \rightarrow \infty} \int_{\Omega_{m}^{i}}\left(\left|\nabla\left(u_{m}^{i}\right)\right|^{2}+\left|u_{m}^{i}\right|^{2}\right) d x=a^{i}, \\
& \lim _{m \rightarrow \infty} \int_{\Omega_{m}^{i}}\left|\nabla u_{m}^{i}\right|^{2}\left|u_{m}^{i}\right|^{2} d x=b^{i}, \\
& \lim _{m \rightarrow \infty} \lambda \int_{\Omega_{m}^{i}}\left|u_{m}^{i}\right|^{p} d x=c^{i} .
\end{aligned}
$$

Then (3.19) reads

$$
a^{i}+2 b^{i}\left(t_{*}^{i}\right)^{2}-c^{i}\left(t_{*}^{i}\right)^{p-2}=0
$$

But from $u_{m}^{i}(x) \in M\left(\Omega_{m}^{i}\right)$ we know that

$$
a^{i}+2 b^{i}-c^{i}=0
$$

Set

$$
h(s)=a^{i}+2 b^{i} s^{2}-c^{i} s^{p-2} .
$$

It is easy to verify that $h(s)$ has only one zero point in $(0,+\infty)$. Taking $(3.20)$ and (3.21) into account, we have $t_{*}^{i}=1$. So (3.13) holds. Moreover, by (3.13), (3.15)-(3.18), we deduce that

$$
\lim _{m \rightarrow \infty} I\left(t_{m}^{i} u_{m}^{i}(y)\right)=\lim _{m \rightarrow \infty} I\left(u_{m}^{i}(x)\right) .
$$

On the other hand, since $I\left(\hat{u}^{i}\right)=\inf _{M\left(\Omega^{i}\right)} I(u)$ and $t_{m}^{i} u_{m}^{i}(y) \in M\left(\Omega^{i}\right)$, we get

$$
I\left(\hat{u}^{i}\right) \leq I\left(t_{m}^{i} u_{m}^{i}(y)\right)
$$

and hence

$$
\lim _{m \rightarrow \infty} I\left(u_{m}^{i}(x)\right) \geq I\left(\hat{u}^{i}\right), \quad i=1,2, \cdots, k+1 .
$$

Thus

$$
c_{k}=\lim _{m \rightarrow \infty} I\left(u_{m}\right)=\lim _{m \rightarrow \infty} \sum_{i=1}^{k+1} I\left(u_{m}^{i}\right) \geq \sum_{i=1}^{k+1} I\left(\hat{u}^{i}\right)=I\left(u_{k}\right) .
$$

Since $u_{k} \in M_{k}$, we have that $c_{k}=I\left(u_{k}\right)$, which means that $c_{k}$ is attained.

Now, we are ready to prove the main result.

Proof of Theorem 1.1. By Lemma 3.1, there exists $u_{k} \in M_{k}$ which attains $c_{k}$. We will prove that $u_{k}$ is indeed a solution to problem (1.3). For convenience, we denote $u:=u_{k}$. Thus we get $k$ nodes: $r_{1}, r_{2}, \cdots, r_{k}, 0<r_{1}<r_{2}<\cdots<r_{k}<+\infty$. Clearly, $u$ satisfies (1.3) in $\left\{x \in \mathbb{R}^{N}:|x| \neq r_{j}, j=1,2, \cdots, k+1\right\}$. We set $r:=|x|$ and treat (1.3) as an ordinary differential equation. To simplify notation we write $u(r)$ instead of $u(|x|)$. We know already that $u$ is of class $C^{2}$ on

$$
E=\left\{r \in(0,+\infty): r \neq r_{j}, j=1,2, \cdots, k\right\}
$$


and satisfies, for $r \in E$,

$$
-\left(1+u^{2}\right)\left(r^{N-1} u^{\prime}\right)^{\prime}=r^{N-1}\left(\lambda|u|^{p-2}-V+\left|u^{\prime}\right|^{2}\right) u,
$$

where ' denotes $\frac{d}{d r}$. To complete the proof, it suffices to show that $u$ satisfies (3.23) for all $r>0$. This is the case if and only if

$$
u_{+}^{\prime}=\lim _{r \searrow r_{j}} u^{\prime}(r)=\lim _{r \succ r_{j}} u^{\prime}(r)=u_{-}^{\prime}, \quad j=1,2, \cdots, k .
$$

In order to get (3.24), we use an indirect argument. Assume that $u_{+}^{\prime} \neq u_{-}^{\prime}$ and set $\rho=r_{j-1}, \sigma=r_{j}, \tau=r_{j+1}$. We may assume that $u \geq 0$ on $[\rho, \sigma], u \leq 0$ on $[\sigma, \tau]$. Now fix $\delta>0(0<\min \{\sigma-\rho, \tau-\sigma\})$ and define $v:[\rho, \tau] \rightarrow \mathbb{R}$ by

$$
v(r)= \begin{cases}u(r), & \text { if }|r-\sigma| \geq \delta, \\ u(\sigma-\delta)+\frac{(r-\sigma+\delta)[u(\sigma+\delta)-u(\sigma-\delta)]}{2 \delta}, & \text { if }|r-\sigma|<\delta .\end{cases}
$$

Clearly, $v$ is continuous on $[\rho, \tau]$. Let $\sigma_{0}=\sigma_{0}(\delta) \in(\sigma-\delta, \sigma+\delta)$ be defined by $v\left(\sigma_{0}\right)=0$. According to (3.1), there exist $\alpha=\alpha(\delta)>0, \beta=\beta(\delta)>0$ such that

$$
\begin{aligned}
& \int_{\rho}^{\sigma_{0}}\left(\left|\alpha v^{\prime}\right|^{2}+V|\alpha v|^{2}+2\left|\alpha v^{\prime}\right|^{2}|\alpha v|^{2}\right) r^{N-1} d r=\lambda \int_{\rho}^{\sigma_{0}}|\alpha v|^{p} r^{N-1} d r, \\
& \int_{\sigma_{0}}^{\tau}\left(\left|\beta v^{\prime}\right|^{2}+V|\beta v|^{2}+2\left|\beta v^{\prime}\right|^{2}|\beta v|^{2}\right) r^{N-1} d r=\lambda \int_{\sigma_{0}}^{\tau}|\beta v|^{p} r^{N-1} d r .
\end{aligned}
$$

Next we define

$$
w(r)= \begin{cases}\alpha v(r), & \rho \leq r \leq \sigma_{0} \\ \beta v(r), & \sigma_{0} \leq r \leq \tau \\ u(r), & \text { otherwise }\end{cases}
$$

hence $w \in M_{k}$. By the definition of $u$, we have

$$
\psi(u) \leq \psi(w)
$$

where

$$
\psi(h)=\int_{\rho}^{\tau}\left(\frac{1}{2}\left(h^{\prime 2}+V h^{2}+h^{2} h^{\prime 2}\right)-\frac{\lambda}{p}|h|^{p}\right) r^{N-1} d r .
$$

Since $|\sqrt{h}|^{p}=h^{\frac{p}{2}}$ is convex for $h>0$, we have

$$
\frac{1}{p}|w|^{p}>\frac{1}{p}|u|^{p}+\frac{w^{2}-u^{2}}{2}|u|^{p-2}, \quad \text { if } u, w>0 .
$$

It follows that

$$
\begin{gathered}
\left\{\int_{\rho}^{\sigma-\delta}+\int_{\sigma+\delta}^{\tau}\right\}\left\{\left(\frac{1}{2}\left(\left|w^{\prime}\right|^{2}+V w^{2}+w^{2}\left|w^{\prime}\right|^{2}\right)-\frac{\lambda}{p}|w|^{p}\right) r^{N-1} d r\right\} \\
\leq\left\{\int_{\rho}^{\sigma-\delta}+\int_{\sigma+\delta}^{\tau}\right\}\left\{\left(\frac{1}{2}\left(\left|w^{\prime}\right|^{2}+V w^{2}+w^{2}\left|w^{\prime}\right|^{2}\right)-\frac{\lambda}{p}|u|^{p}\right.\right. \\
\left.\left.-\frac{\lambda}{2}\left(w^{2}-u^{2}\right)|u|^{p-2}\right) r^{N-1} d r\right\} .
\end{gathered}
$$


On the other hand,

$$
\begin{aligned}
& \int_{\sigma-\delta}^{\sigma+\delta}\left(\frac{1}{2}\left(\left|w^{\prime}\right|^{2}+V w^{2}+w^{2}\left|w^{\prime}\right|^{2}\right)-\frac{\lambda}{p}|w|^{p}\right) r^{N-1} d r \\
\leq & \int_{\sigma-\delta}^{\sigma+\delta}\left(\frac{1}{2}\left(\left|w^{\prime}\right|^{2}+V w^{2}+w^{2}\left|w^{\prime}\right|^{2}\right)-\frac{\lambda}{p}|w|^{p}+\frac{\lambda}{p}|u|^{p}-\frac{\lambda}{p}|u|^{p}+\frac{\lambda}{2}|u|^{p}\right) r^{N-1} d r .
\end{aligned}
$$

By the definition of $u$, we have

$$
\int_{\rho}^{\tau}\left(\left|u^{\prime}\right|^{2}+V u^{2}+2 u^{2}\left|u^{\prime}\right|^{2}\right) r^{N-1} d r=\lambda \int_{\rho}^{\tau}|u|^{p} r^{N-1} d r .
$$

Thus, combining (3.25)-(3.28) we obtain

$$
\begin{aligned}
& \psi(w) \leq \psi(u) \\
+ & \left\{\int_{\rho}^{\sigma-\delta}+\int_{\sigma+\delta}^{\tau}\right\}\left\{\left(\frac{1}{2}\left(\left|w^{\prime}\right|^{2}+V w^{2}+w^{2}\left|w^{\prime}\right|^{2}+u^{2}\left|u^{\prime}\right|^{2}\right)-\frac{\lambda}{2} w^{2}|u|^{p}\right) r^{N-1} d r\right\} \\
+ & \int_{\sigma-\delta}^{\sigma+\delta}\left(\frac{1}{2}\left(\left|w^{\prime}\right|^{2}+V w^{2}+w^{2}\left|w^{\prime}\right|^{2}+u^{2}\left|u^{\prime}\right|^{2}\right)-\frac{\lambda}{p}|w|^{p}+\frac{\lambda}{p}|u|^{p}\right) r^{N-1} d r .
\end{aligned}
$$

Using (3.23), we see that

$$
\begin{aligned}
& \int_{\rho}^{\sigma-\delta}\left(\frac{1}{2}\left(\left|w^{\prime}\right|^{2}+V w^{2}+w^{2}\left|w^{\prime}\right|^{2}+u^{2}\left|u^{\prime}\right|^{2}\right)-\frac{\lambda}{2} w^{2}|u|^{p}\right) r^{N-1} d r \\
= & \frac{\alpha^{2}}{2} \int_{\rho}^{\sigma-\delta}\left(\left|u^{\prime}\right|^{2}+V u^{2}+\alpha^{2} u^{2}\left|u^{\prime}\right|^{2}-\lambda|u|^{p}\right) r^{N-1} d r+\frac{1}{2} \int_{\rho}^{\sigma-\delta} u^{2}\left|u^{\prime}\right|^{2} r^{N-1} d r \\
= & \frac{\alpha^{2}}{2} \int_{\rho}^{\sigma-\delta}\left(\left|u^{\prime}\right|^{2}+V u^{2}+2 u^{2}\left|u^{\prime}\right|^{2}-\lambda|u|^{p}\right) r^{N-1} d r+\frac{\left(\alpha^{2}-1\right)^{2}}{2} \int_{\rho}^{\sigma-\delta} u^{2}\left|u^{\prime}\right|^{2} r^{N-1} d r \\
= & \frac{\alpha^{2}}{2}\left[u(\sigma-\delta)+u^{3}(\sigma-\delta)\right](\sigma-\delta)^{N-1} u^{\prime}(\sigma-\delta)+\frac{\left(\alpha^{2}-1\right)^{2}}{2} \int_{\rho}^{\sigma-\delta} u^{2}\left|u^{\prime}\right|^{2} r^{N-1} d r .
\end{aligned}
$$

Since $u(\sigma)=0,\left.\left(r^{N-1} u^{\prime}\right)^{\prime}\right|_{r=\sigma}=0$ by (3.23), we obtain

$$
\begin{aligned}
& u(\sigma-\delta)=-\delta u_{-}^{\prime}+o(\delta), \\
& (\sigma-\delta)^{N-1} u^{\prime}(\sigma-\delta)=\sigma^{N-1} u_{-}^{\prime}+o(\delta) .
\end{aligned}
$$

By (3.1), it is easy to verify that

$$
\lim _{\delta \rightarrow 0} \alpha(\delta)=\lim _{\delta \rightarrow 0} \beta(\delta)=1 .
$$

It follows from (3.30)-(3.32) that

$$
\begin{aligned}
& \int_{\rho}^{\sigma-\delta}\left(\frac{1}{2}\left(\left|w^{\prime}\right|^{2}+V w^{2}+w^{2}\left|w^{\prime}\right|^{2}+u^{2}\left|u^{\prime}\right|^{2}\right)-\frac{\lambda}{2} w^{2}|u|^{p}\right) r^{N-1} d r \\
= & \frac{\alpha^{2}}{2}(\sigma-\delta)^{N-1} u^{\prime}(\sigma-\delta) u(\sigma-\delta)+\frac{\left(\alpha^{2}-1\right)^{2}}{2} \int_{\rho}^{\sigma-\delta} u^{2}\left|u^{\prime}\right|^{2} r^{N-1} d r \\
= & -\frac{\sigma^{N-1}}{2}\left(u_{-}^{\prime}\right)^{2} \delta+o(\delta) .
\end{aligned}
$$


Similarly, we can prove that

$$
\int_{\sigma+\delta}^{\tau}\left(\frac{1}{2}\left(\left|w^{\prime}\right|^{2}+V w^{2}+w^{2}\left|w^{\prime}\right|^{2}+u^{2}\left|u^{\prime}\right|^{2}\right)-\frac{\lambda}{2} w^{2}|u|^{p}\right) r^{N-1} d r=-\frac{\sigma^{N-1}}{2}\left(u_{+}^{\prime}\right)^{2} \delta+o(\delta) .
$$

On the other hand, it is not difficult to check that

$$
\begin{aligned}
& \int_{\sigma-\delta}^{\sigma+\delta}\left(\frac{1}{2}\left(V w^{2}+w^{2}\left|w^{\prime}\right|^{2}+u^{2}\left|u^{\prime}\right|^{2}\right)-\frac{\lambda}{p}|w|^{p}+\frac{\lambda}{p}|u|^{p}\right) r^{N-1} d r=o(\delta) . \\
& \frac{1}{2} \int_{\sigma-\delta}^{\sigma+\delta}\left|w^{\prime}\right|^{2} r^{N-1} d r=\frac{1}{2} \int_{\sigma-\delta}^{\sigma+\delta}\left|v^{\prime}\right|^{2} r^{N-1} d r+o(\delta) \\
&=\frac{[u(\sigma+\delta)-u(\sigma-\delta)]^{2}}{8 \delta^{2}}\left(\frac{(\sigma+\delta)^{N}}{N}-\frac{(\sigma-\delta)^{N}}{N}\right)+o(\delta) \\
&=\frac{\sigma^{N-1}}{4}\left(u_{+}^{\prime}+u_{-}^{\prime}\right)^{2} \delta+o(\delta) .
\end{aligned}
$$

Now combining (3.29) and (3.33)-(3.36), we deduce that

$$
\psi(w) \leq \psi(u)-\frac{\sigma^{N-1}}{4}\left(u_{+}^{\prime}-u_{-}^{\prime}\right)^{2} \delta+o(\delta) .
$$

This implies that $\psi(w)<\psi(u)$ for $\delta>0$ small enough, which contradicts the fact that $\psi(u) \leq \psi(w)$.

As a result, we complete the proof.

Acknowledgement. The authors would like to thank the referees for careful reading of the details, valuable comments and suggestions, including pointing out the reference [27]. Deng and Peng were supported by the funds from NSFC(11071092, 11071094). Peng was also supported by NCET(07-0350) and the PhD specialized grant of the Ministry of Education of China (20100144110001).

\section{REFERENCES}

[1] A. Ambrosetti and P.H. Rabinowitz, Dual variational methods in critical point theory and applications, J. Funct. Anal., 14, 349-381, 1973.

[2] T. Bartsch and M. Willem, Infinitely many radial solutions of a semilinear elliptic problem on $\mathbb{R}^{N}$, Arch. Ration. Mech. Anal., 124, 261-276, 1993.

[3] T. Bartsch and M. Willem, Infinitely many nonradial solutions of a Euclidean scalar field equation, J. Funct. Anal., 117, 447-460, 1993.

[4] F.G. Bass and N.N. Nasanov, Nonlinear electromagnetic-spin waves, Phys. Rep., 189, 165223,1990

[5] J.M. Bezerra do Ó, O.H. Miyagaki, and S.H.M. Soares, Soliton solutions for quasilinear Schrödinger equations with critical growth, J. Diff. Eqn., 248, 722-744, 2010.

[6] H. Brezis and E. Lieb, A relation between pointwise convergence of function and convergence of functional, Proc. Amer. Math. Soc., 88, 486-490, 1983.

[7] H. Berestycki and P.L. Lions, Nonlinear scalar field equations, I: Existence of a ground state, Arch. Ration. Mech. Anal., 82, 313-345, 1983.

[8] H. Brandi, C. Manus, G. Mainfray, T. Lehner and G. Bonnaud, Relativistic and ponderomotive self-focusing of a laser beam in a radially inhomogeneous plasma, Phys. Fluids B, 5, 3539$3550,1993$. 
[9] L. Brizhik, A. Eremko, B. Piette, and W.J. Zakrzewski, Static solutions of a D-dimensional modified nonlinear Schrödinger equation, Nonlinearity, 16, 1481-1497, 2003.

[10] L. Brüll and H. Lange, Solitary waves for quasilinear Schrödinger equations, Exposition Math., 4, 279-288, 1986.

[11] D. Cao and X. Zhu, On the existence and nodal character of semilinear elliptic equations, Acta. Math. Sci., 8, 345-359, 1988.

[12] X.L. Chen and R.N. Sudan, Necessary and sufficient conditions for self-focusing of short ultraintense laser pulse in underdense plasma, Phys. Rev. Lett., 70, 2082-2085, 1993.

[13] M. Colin and L. Jeanjean, Solutions for a quasilinear Schrödinger equation: A dual approach, Nonlinear Anal. TMA., 56, 213-226, 2004.

[14] M. Conti, L. Merizzi, and S. Terracini, Radial solutions of superlinear equations on $\mathbb{R}^{N}$. I. A global variational approach, Arch. Ration. Mech. Anal., 153, 291-316, 2000.

[15] S. Cuccagna, On instability of excited states of the nonloinear Schrödinger equation, Physica D, 238, 38-54, 2009.

[16] A. De Bouard, N. Hayashi, and J. Saut, Global existence of small solutions to a relativistic nonlinear Schrödinger equation, Commun. Math. Phys., 189, 73-105, 1997.

[17] A. Floer and A. Weinstein, Nonspreading wave packets for the cubic Schrödinger equation with a bounded potential, J. Funct. Anal., 69, 397-408, 1986.

[18] D. Gilbarg and N.S. Trudinger, Elliptic Partial Differential Equations of Second Order, Springer-Verlag, Berlin-New York, 1998.

[19] R.W. Hasse, A general method for the solution of nonlinear soliton and kink Schrödinger equations, Z. Phys. B, 37, 83-87, 1980.

[20] A.M. Kosevich, B.A. Ivanov, and A.S. Kovalev, Magnetic solitons, Phys. Rep., 94, 117-238, 1990.

[21] S. Kurihara, Large-amplitude quasi-solitons in superfluid films, J. Phys. Soc. Japan, 50, 32623267, 1981.

[22] E. Laedke, K. Spatschek, and L. Stenflo, Evolution theorem for a class of perturbed envelope soliton solutions, J. Math. Phys., 24, 2764-2769, 1983.

[23] H. Lange, M. Poppenberg, and H. Teismann, Nash-Moser methods for the solution of quasilinear Schrödinger equations, Commun. Par. Diff. Eq., 24, 1399-1418, 1999.

[24] J. Liu and Z. Wang, Soliton solutions for quasilinear Schrödinger equations. I., Proc. Amer. Math. Soc., 131, 441-448, 2003.

[25] J. Liu, Y. Wang, and Z. Wang, Soliton solutions for quasilinear Schrödinger equations. II, J. Diff. Eq., 187, 473-493, 2003.

[26] J. Liu, Y. Wang, and Z. Wang, Solutions for quasilinear Schrödinger equations via the Nehari method, Commun. Par. Diff. Eq., 29, 879-901, 2004.

[27] J. Liu and Z. Wang, Symmetric solutions to a modified nonlinear Schrödinger equation, Nonlinearity, 21, 121-133, 2008.

[28] V.G. Makhankov and V.K. Fedyanin, Nonlinear effects in quasi-one-dimensional models and condensed matter theory, Phys. Rep., 104, 1-86, 1984.

[29] Z. Nehari, Characteristic values associated with a class of non-linear second-order differential equations, Acta Math., 105, 141-175, 1961.

[30] M. Poppenberg, Smooth solutions for a class of fully nonlinear Schrödinger type equations, Nonlinear Anal. TMA., 45, 723-741, 2001.

[31] M. Poppenberg, On the local well posedness of quasilinear Schrödinger equations in arbitrary space dimension, J. Diff. Eq., 172, 83-115, 2001.

[32] M. Poppenberg, K. Schmitt, and Z. Wang, On the existence of soliton solutions to quasilinear Schrödinger equations, Calc. Var. Par. Diff. Eq., 14, 329-344, 2002.

[33] G.R.W. Quispel and H.W. Capel, Equation of motion for the Heisenberg spin chain, Phys. A, 110, 41-80, 1982.

[34] P.H. Rabinowitz, On a class of nonlinear Schrödinger equations, Z. Angew. Math. Phys., 43, 270-291, 1992.

[35] B. Ritchie, Relativistic self-focusing and channel formation in laser-plasma interactions, Phys. Rev. E, 50, 687-689, 1994.

[36] W.A. Strauss, Existence of solitary waves in higher dimensions, Commun. Math. Phys., 55, 149-162, 1977.

[37] T. Weth, Energy bounds for entire nodal solutions of autonomous superlinear equations, Calc. Var. Par. Diff. Eq., 27, 421-437, 2006. 